\title{
The new concept of power transmission to the entomopter wings
}

\author{
Wojciech Sochacki ${ }^{1} \cdot$ Dawid Cekus $^{1}$ (1)
}

Received: 14 December 2019/Revised: 7 May 2020 / Accepted: 4 June 2020 / Published online: 12 June 2020

(C) The Author(s) 2020

\begin{abstract}
The aim of this study is to discuss the design of the mechanism used for power transmission to the entomopter wings in order to perform a flapping motion and control the angle of attack of the wings. The study presents a kinematic diagram and a simulation model obtained in SolidWorks for the proposed mechanism, which includes a slotted link mechanism and a slider mechanism (with bilateral slider) that actuates the rocking lever of the wing. The simulation model allowed for observation of the system work and verification of the adopted kinematic assumptions. The comparative analysis showed that trajectories obtained from the both models are very similar. The correct operation of the proposed solution has been demonstrated by building a prototype of the mechanism and conducting experimental research. In the case of the application of the solution presented for the real object it is sufficient to choose the system parameters in order to properly reflect the living organism. The proposed mechanism is characterized by simplicity and offers opportunity for miniaturization while ensuring reliable work at reduced demand for power to drive the mechanism. A technological advantage of the presented solution is the use of only one component in order to perform flapping wing motion and change the angle of attack of the entomopter.
\end{abstract}

Keywords Entomopter $\cdot$ Flapping $\cdot$ Angle of attack $\cdot$ Power transmission mechanism $\cdot$ Wing

\section{Introduction}

Potential applications of entomopters (flying miniature robots) include observation, control and supervision of e.g. building investments, internal monitoring of pipelines (especially those with small diameters), identification of damages caused by natural disasters, battlefield surveillance and inspection of inaccessible regions (forestry, environmental protection), geodetics etc.

The mechanism of power transmission to the entomopter wings has been used worldwide for many years. Various scientific and research centres from various countries have presented plethora of solutions concerning forced movement of

Electronic supplementary material The online version of this article (https://doi.org/10.1007/s12213-020-00135-2) contains supplementary material, which is available to authorized users.

Dawid Cekus

cekus@imipkm.pcz.pl

Wojciech Sochacki

sochacki@imipkm.pcz.pl

1 Department of Mechanics and Machine Design Fundamentals, Czestochowa University of Technology, Dabrowskiego 73, Częstochowa, Poland micro air vehicles. The solutions described in the literature concerned the flapping wing movement and changing the angle of attack of wings.

Description of a number of solutions of various types of mechanisms of power transmission to the wings of a flying minirobot included in the article [1]. The proposed mechanisms are primarily designed to reproduce the complicated movement of the wings of a real insect without considering folding their up [2]. Among the studies that have analysed the kinematics of movement and the mechanism of mechanical power transmission into entomopter wings are publications [3-32]. A differential mechanism [3], a crank-and-slider mechanism and a slider-rocker mechanism [4-7], a cam mechanism [8], a four-bar linkage [9-16] or a Scotch yoke [17-19] were used to power transmission. The power transmission system cooperating with the Weis-Fogh mechanism is presented in [20]. The power transmission systems can also be supported by piezoelectric elements. In this case, the basic movement of the wings is realized in the classic way, but with the help of mechanical transmission with periodic modification of the trajectory using the piezoelectric elements [21-23]. In turn, the use of the most advanced projects of the resonant drive is shown in the works [24-26]. A physical model for a micro air vehicle with Flapping Rotary Wings (FRW) is investigated in paper [27]. The comprehensive MAV concepts 
Fig. 1 Kinematic diagram of the mechanism of power transmission to the entomopter wing

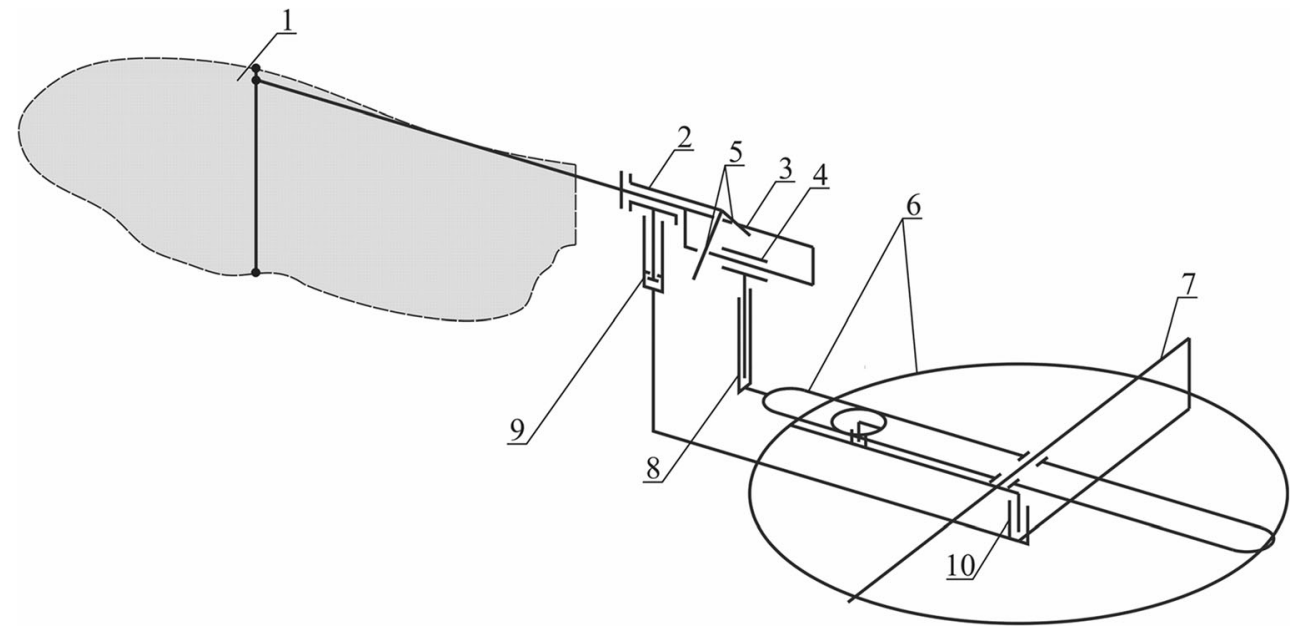

carried out in various research centres, in which the drive and the flapping wing motion were analyzed, were presented in the works [28-32].

The solutions for transmission of power to the wings of flying objects that mimic the insect's wing motion have been also presented in numerous patents [33-40].

In the present study, we presented the mechanism of power transmission to entomopter wings by means of a simple slotted-link-slider mechanism. Insect's wing motion is composed (in simplification) from the flapping motion and the motion connected with changes in the angle of attack of the wing. These two movements occur in various planes in specific wing positions. Changes in the angle of attack of the wing occur in extreme positions of the flapping wing. The mechanism proposed in this study enables to produce this movement in a micro air vehicle (MAV). The idea of the solution is to use the slider mechanism with bilateral slider which, combined with the rocking lever of the wing, allows sequential changes of the mechanical wing in specific phases of its motion.

\section{The concept of mechanism}

The proposed mechanism was created in stages, i.e. first a kinematic model of the system was developed, and then a

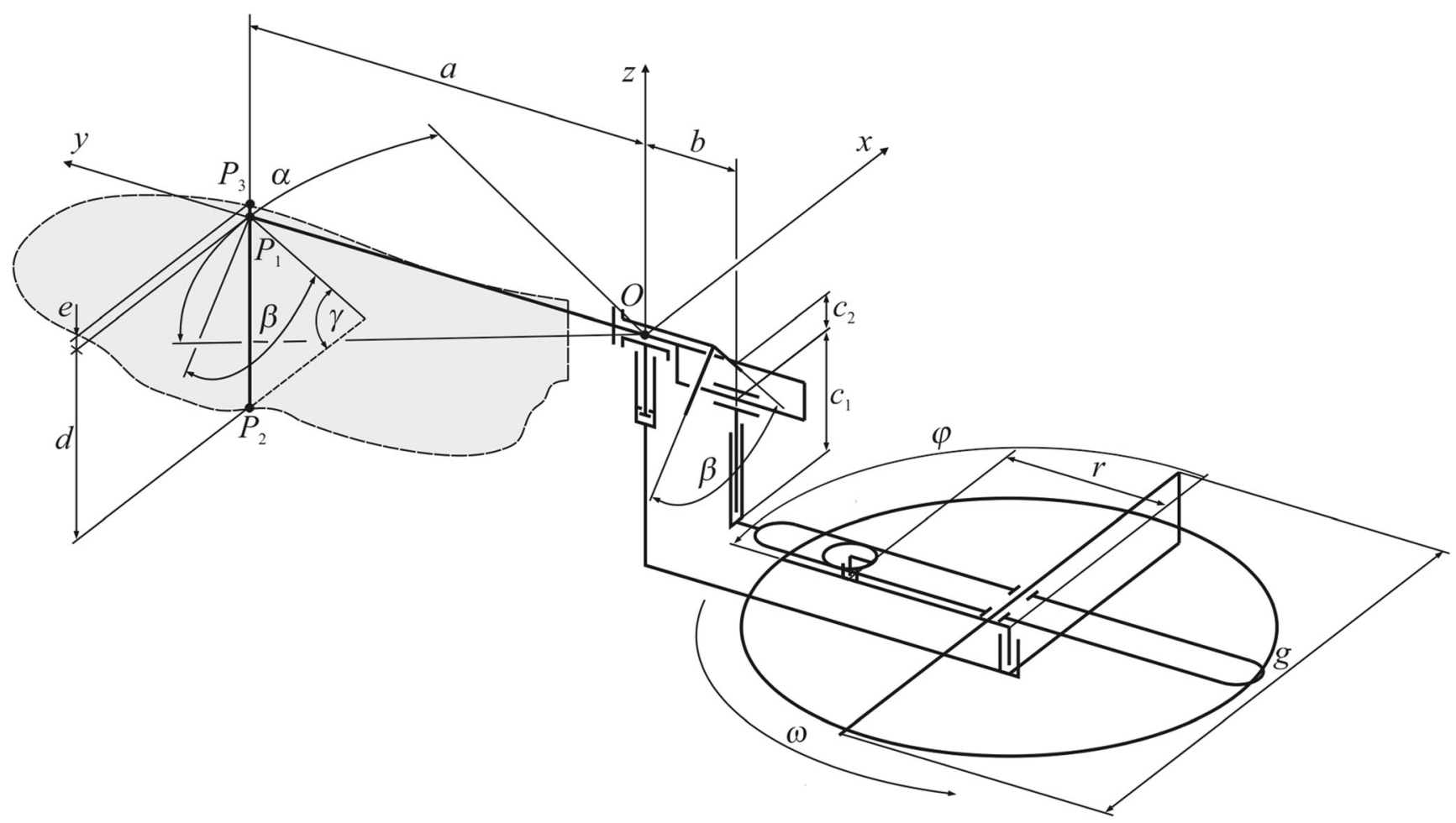

Fig. 2 Parametrized kinematic diagram of the mechanism of power transmission to the entomopter wing 
Fig. 3 Model of the mechanism

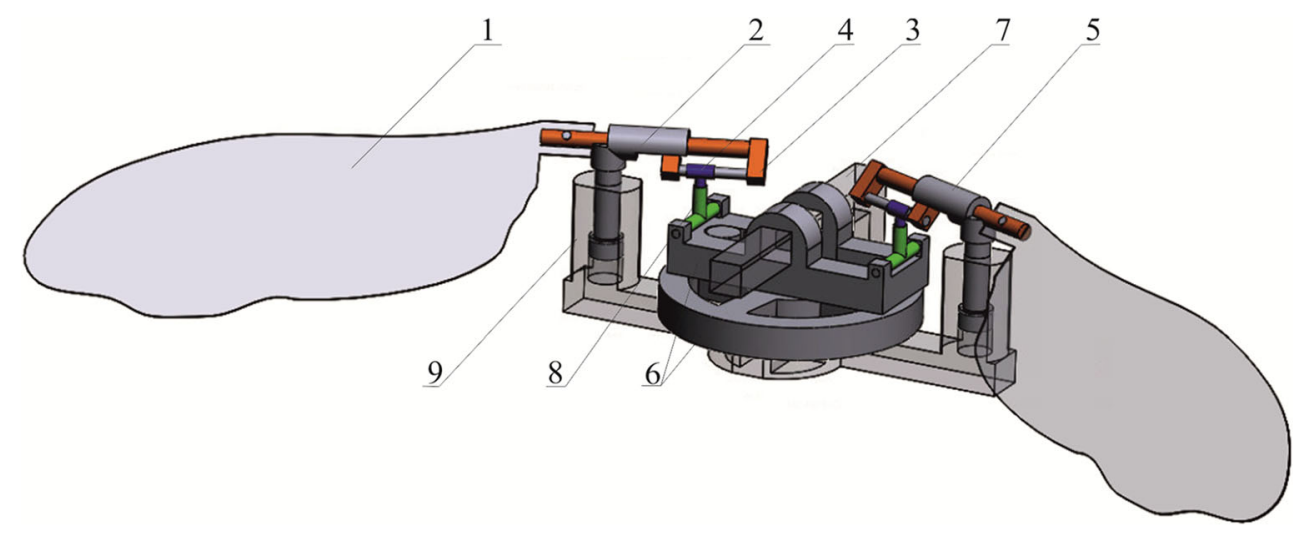

geometric simulation model was prepared in the SolidWorks program. The simulation model allowed for observation of the system work and verification of the adopted kinematic assumptions.

\subsection{Kinematic model}

Kinematic model (for one wing) of the proposed mechanism was shown in Fig. 1. It is composed of: 1 - entomopter wing, 2 - rocking lever guide with limiters of the angle of attack 5, 3 wing rocking lever, 4 - bilateral slide, 6 - slotted-link mechanism, 7 - slotted-link mechanism guide, 8 - bushing and guide of the bilateral slide, 9 - wing rocking level guide bushing, 10 slotted-link mechanism bushing.

The following symbols were used in the kinematic diagram to describe mechanism motion sequence (Fig. 2): $O$ - origin of the coordinate system, $P_{1}, P_{2}$, $P_{3}$ - selected points on the wing used to describe wing motion ( $P_{1}$ - point on the $x y$-plane), $a$ - distance of the point $P_{1}$ from the origin of the coordinate system, $b$ distance from the centre of the bilateral slider guide to the origin of the coordinate system, $c_{1}$ - bilateral slider height, $c_{2}$ - wing rocking lever height, $d$ and $e$ - distances of points $P_{2}$ and $P_{3}$ from the point $P_{1}, \alpha$ - angle of flapping motion, $\beta$ - angle of wing rotation, $\gamma$ - angle of attack, $\varphi$ - angle of the position of the driving wheel of the slotted-link mechanism, $\omega$ - angular velocity, $r$ - radius of the driving wheel of the slotted-link mechanism.

Position of the characteristic points $P_{1}, P_{2}$ and $P_{3}$, during the mechanism work can be described by means of the following formulae:

$P_{1 x}=a \sin (-\alpha / 2)$,

$P_{1 y}=a \cos (\alpha / 2)$,

$P_{1 z}=0$,

$P_{2 x}=P_{1 x}+d \sin (\beta / 2) \cos (\alpha / 2)$,

$P_{2 y}=P_{1 y}+d \sin (\beta / 2) \sin (\alpha / 2)$,

$P_{2 z}=P_{1 z}-d \cos (\beta / 2)$,

$P_{3 x}=P_{1 x}-e \sin (\beta / 2) \cos (\alpha / 2)$,

$P_{3 y}=P_{1 x}-e \sin (\beta / 2) \sin (\alpha / 2)$,

$P_{3 z}=P_{1 z}+e \cos (\beta / 2)$.

The value of the parameters $a, d$ and $e$ (dependent of the wing type) are constants and do not change during mechanism operation. The system motion is defined by value of angles $\alpha$ and $\beta$, which depend on the value of angle $\varphi$. The limiting value of angle $\alpha$, which depends on the radius $(r)$ and distance (b) between the bushing of the bilateral slider guide and the bushing of the rocking lever guide can be described by:

$\alpha=2 \arctan (r / b)$.
Fig. 4 Limiters of the rotational (rocking) motion for the rocking lever of the wing
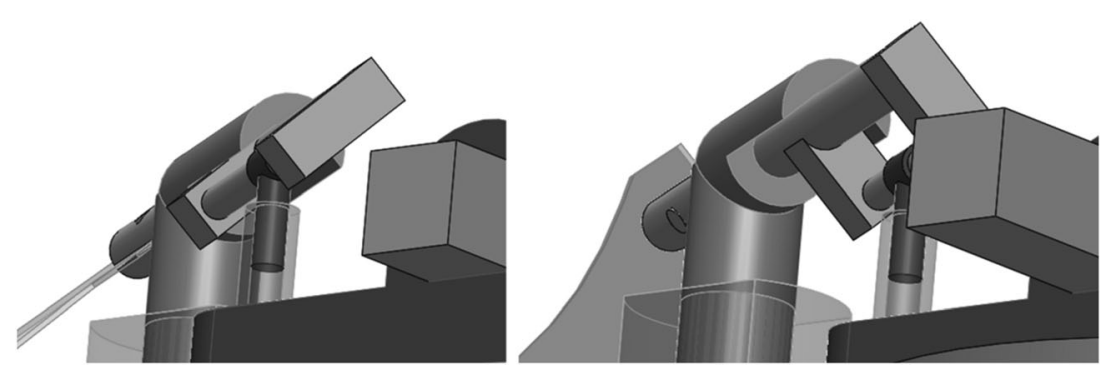


\section{a}

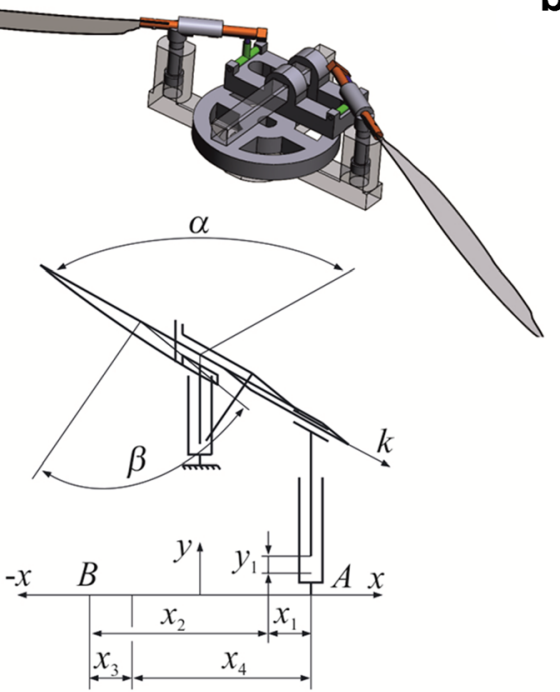

C

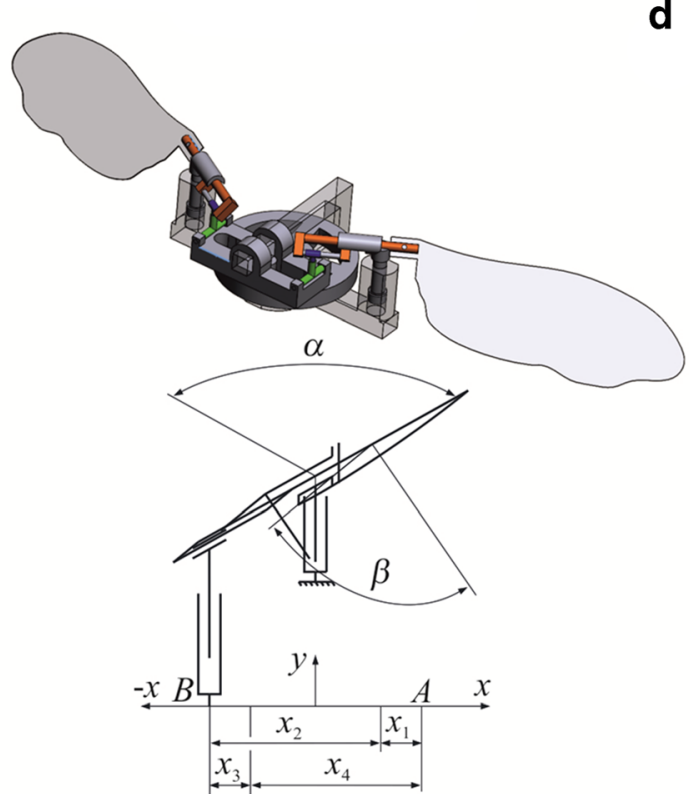

b

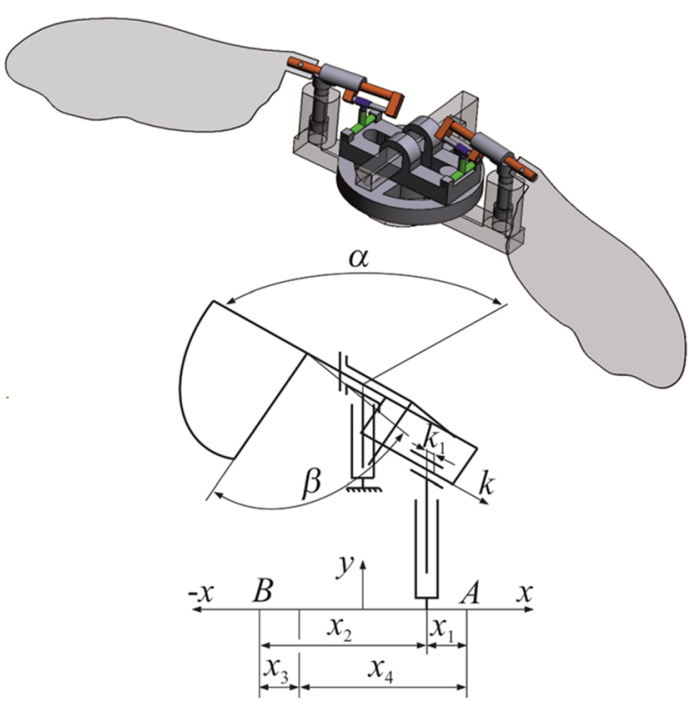

d

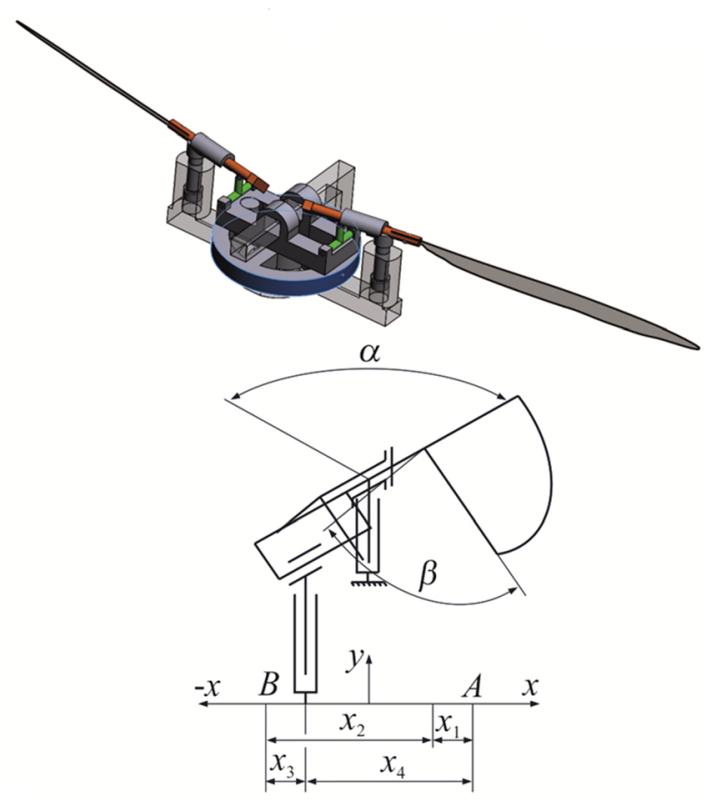

Fig. 5 A full sequence of entomopter wing motion (changes in the angle of wing rotation and flapping angle)

The limiting value of the angle $\beta$ depends on the design of the wing lever guide. Time to reach maximal values depends on the parameter $c_{2}$ (the higher value of the parameter, the longer time).

\subsection{Structural model and the working principle}

A structural model for the proposed mechanism was developed based on the adopted kinematic that concerned the implementation of such a movement of the mechanism, which as a consequence will force the movement of the wings of the model corresponding to the movement of the wings of the real insect.
A solid parametric model was construed using the SolidWorks software [41]. The model (similar to the kinematic design) is composed of the following components: wing 1, rocking lever guide 2 , rocking lever 3, bilateral slider 4, limiters of the angle of wing rotation 5 (adequately shaped guide 2), slotted-link mechanism 6 , slotted-link mechanism 7, bushing and guide of the bilateral slider 8, and guide bushing 9 .

Operation of the mechanism used in the model occurs as follows: the battery-powered electric motor (not shown in Fig. 3) drives the driving wheel of the slotted-link mechanism 6 , with the slotted-link moves along the guide 7 . The slotted link is connected with bushings and bilateral slider guides 8 , on which bilateral slides 4 are fixed. Lower parts of sliders moving in bushings and guides can perform both rotational 
a

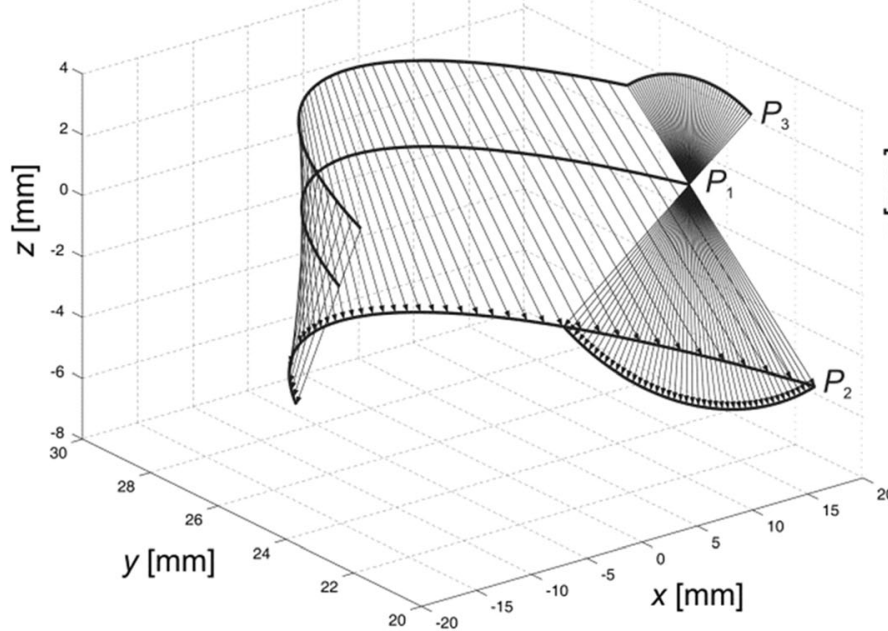

b

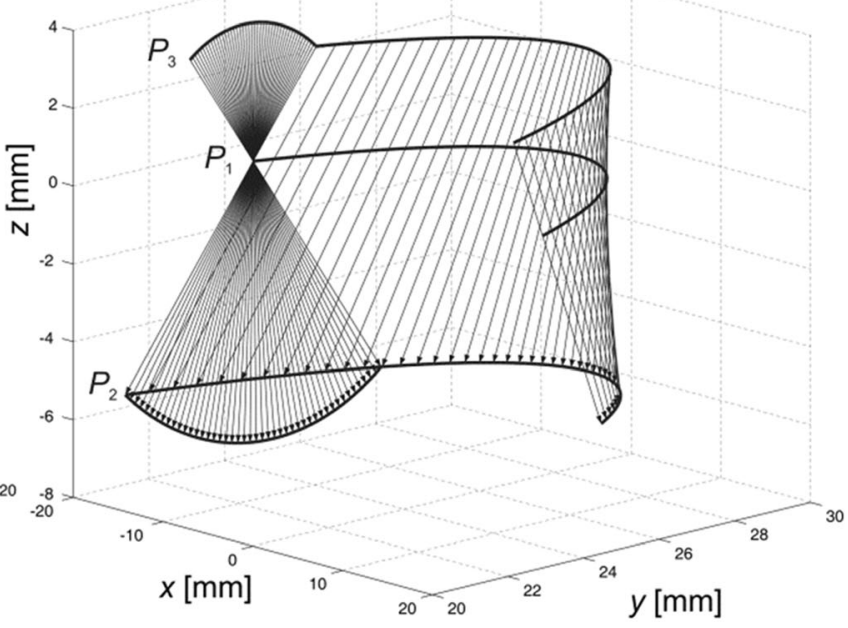

Fig. 7 Location of the characteristic points $P_{1}, P_{2}$ and $P_{3}$ in the isometric view during flapping (downstroke (a) and upstroke (b)) and changes in the angle of wing rotation

and sliding movements. Upper parts of bilateral sliders work together with wings' rocking lever guides 3. During the work of the mechanism, the slider performs four movements. Basic movement (reciprocating), resulting from the movement of the slotted-link mechanism and two relative movements, i.e. extension and rotation in the bushing and guide 8. These movements also lead to moving the slider on the rocking lever guide. The rocking lever of the wing moves in the guide 2 , which is nested in the bushing 9 and performs partially rotational motion in the range of changes of flapping motion $\alpha$. The rocking lever performs limited rotational movement with respect to the guide in the range of the angle of wing rotation $\beta$. The range of motion of the rocking lever defined by the angle $\beta$ is limited by the lever motion limiters. The limiters were obtained by the matching shape of the rocking lever guide (see Fig. 4). Entomopter wings are attached to the rocking levers of the wings located on the other side of the guide.

The described connections of the mechanism components and their movements force the specific movement of the wings. In the beginning of the motion, started from the turning points $A$ and $B$ (see Fig. 5) of the slotted-link mechanism, the
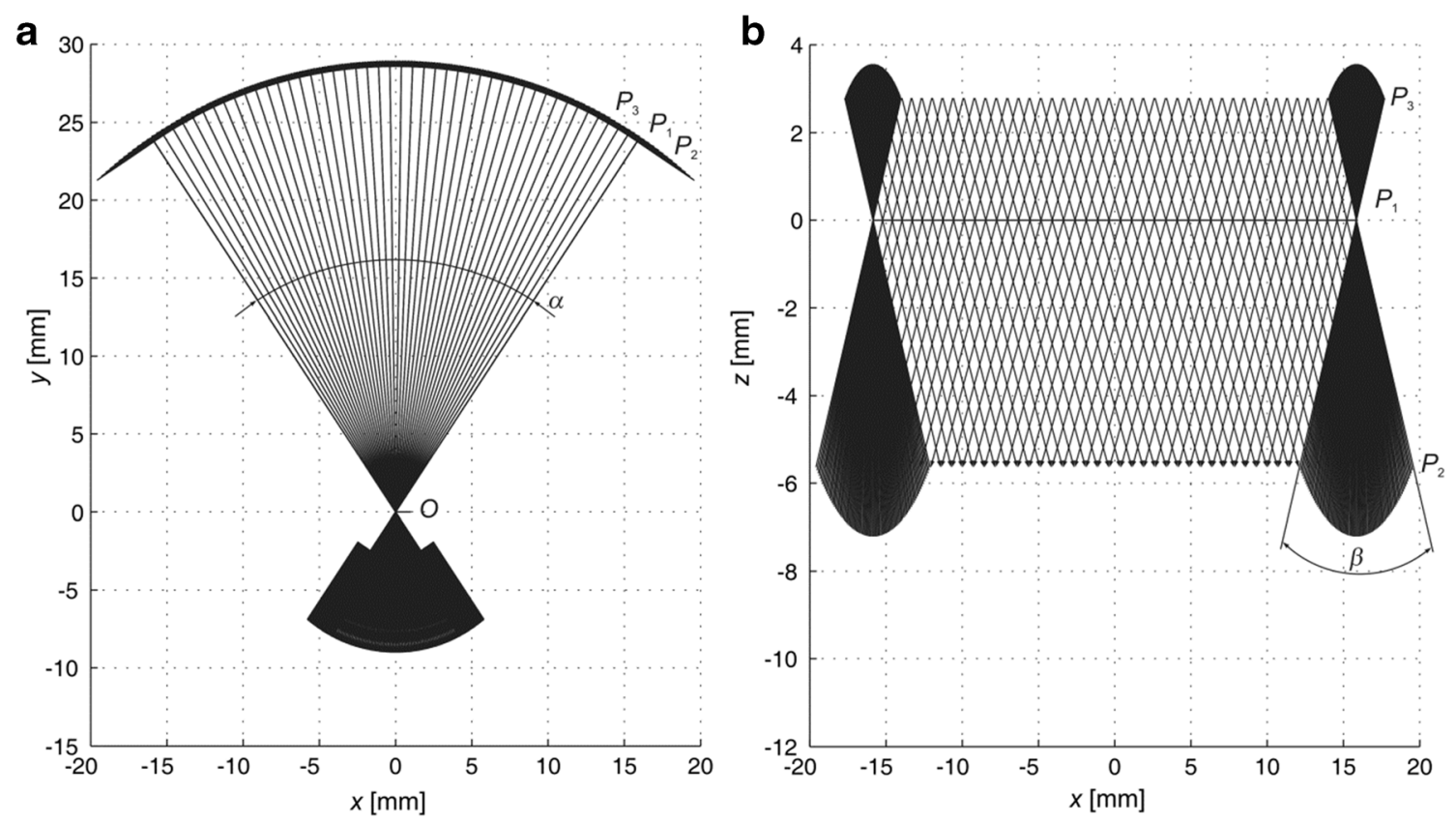

Fig. 8 Location of the characteristic points $P_{1}, P_{2}$ and $P_{3}$ and ranges of angles $\alpha$ and $\beta$ : projections onto the $x y$ plane (a) and $x z$ plane (b) 
Fig. 6 Changes in angles $\alpha$ and $\beta$ during the mechanism work (changes in the angle $\varphi$ )

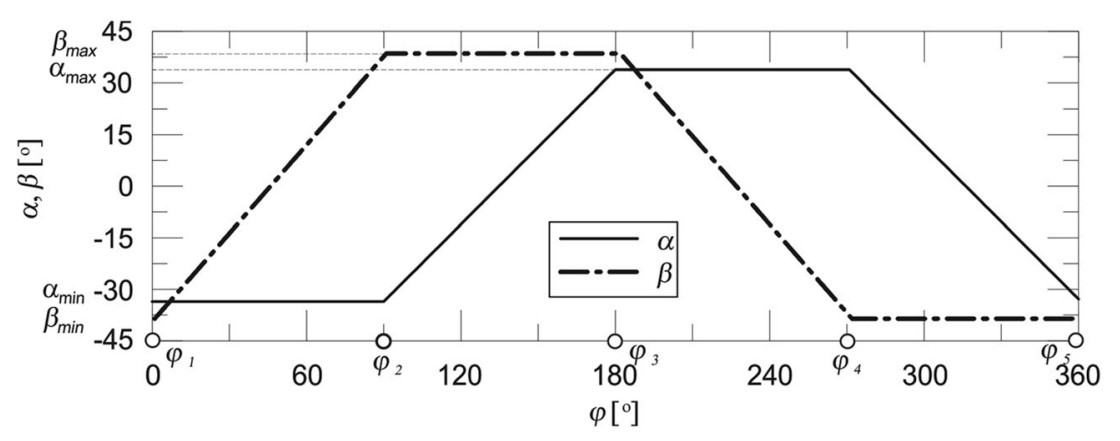

wing first perform the change of the angle of wing rotation $\beta$ and next the flapping motion (change in the angle $\alpha$ ). A full sequence of motion mechanism was illustrated in Fig. 5a-d.

These figures present a simplified form of the slottedlink mechanism and its motion as $x$ and $-x$ displacement of the bushing and guide of the bilateral slider 8 . As the mechanism works, bilateral slider 4 moves in the guide 8 in the range of value $y_{1}$. The rocking lever of the wing 3 rests on the limiter 5 .

Further movement of the slotted-link $x$ leads to the flapping wing motion (change in the angle $\alpha$ by the value of $\alpha / 2$ ). This motion is continued until the slotted link reaches the extreme location (moving the slotted link to the point $A$, see Fig. 5a). In the point $A$, the slotted link switches the direction of motion from $x$ to $-x$ and the returning motion of the slotted link begins. In the first phase of the movement (slotted link movement by the value $x_{1}$ ), it causes the change in the angle of wing rotation by the value $\beta$. The rocking lever rests on the opposite limiter and the flapping motion is started (change in the angle $\alpha$ ) with the motion of the slotted link moving by $x_{2}$ until the second turning point $B$ is reached (Fig. $5 \mathrm{c}$ ).

The change in the direction of the motion of the slotted link mechanism from $-x$ to $x$ occurs in point $B$. During the mechanism movement by $x_{3}$ the angle of wing rotation changes by the value of $\beta$ (see Fig. $5 d$ ).
This represents the starting point of the flapping motion until the mechanism reaches the point $A$. In this point, the motion sequence of the mechanism is completed, and, consequently the wing motion sequence also ends (change in the angle of wing rotation $\beta$ and flapping angle $\alpha$ ) both in $x$ and $-x$ directions. Next, the sequence of motion and changes in angles is repeated again.

A technological advantage of the presented solution is the use of only one component between the slottedlink mechanism and the rocking lever of the wing in the form of a bilateral slider in order to perform flapping wing motion and change the angle of wing rotation of the entomopter. The simplicity of the solution allows for the independent work of the mechanism, reduction in power consumed to drive the mechanism and opportunities for substantial miniaturization of the design.

\section{Example results}

During a flight, insects perform a flapping motion over various ranges of the angle $\alpha$. Example values of the wing flapping angle were presented in the study [18]. As results from the data presented in this study, the angles range from $56^{\circ}$ to $91^{\circ}$. Furthermore, the typical

Fig. 9 Geometrical wing model with characteristic points

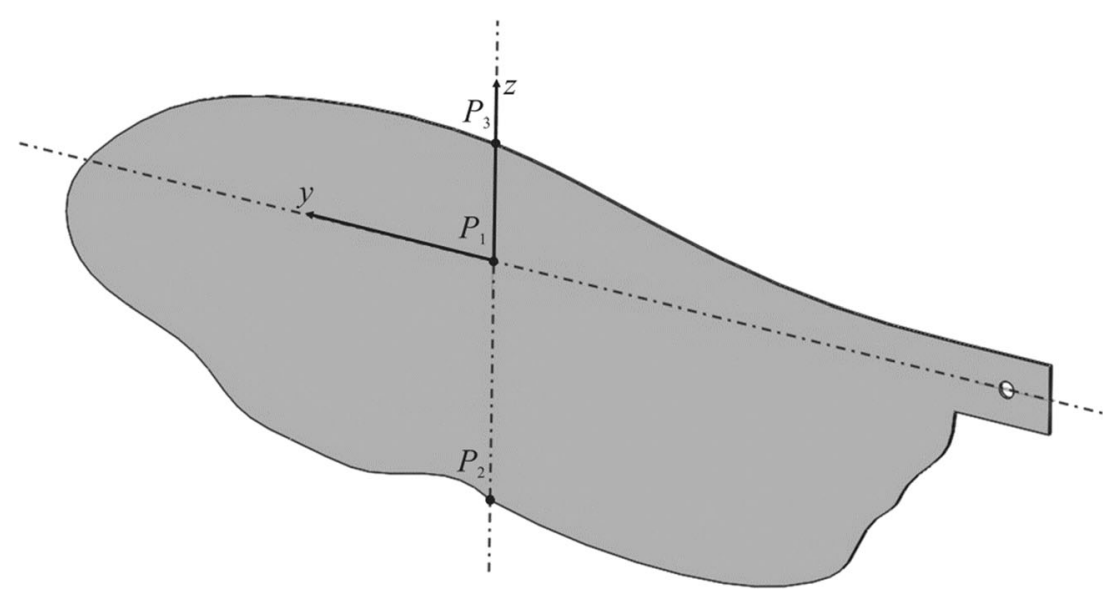


Fig. 10 Trajectory of the characteristic points during the mechanism operation

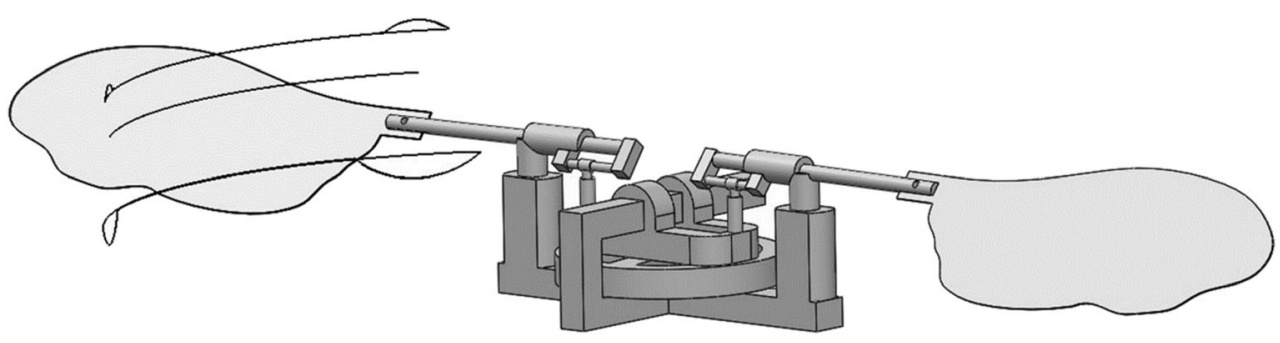

angle of attack at $70 \%$ wingspan ranges from $\gamma=25^{\circ}$ to $\gamma=45^{\circ}$ in hovering insects.

The following values of the parameters were adopted in the model (see Fig. 2): $a=28.57 \mathrm{~mm}, b=7.4 \mathrm{~mm}, c_{1}=4.9 \mathrm{~mm}$, $c_{2}=1.6 \mathrm{~mm}, d=7.21 \mathrm{~mm}, e=3.56 \mathrm{~mm}, r=4.74 \mathrm{~mm}$ and $\beta=$ $77.11^{\circ}$, and, consequently, using the adopted relationship (4), $\alpha=67^{\circ}$.

\subsection{Analytical solution}

Based on the parameters obtained from the developed geometrical model (see Fig. 3) and the relationships (1-3), we determined locations of the characteristic points of the wing $\left(P_{1}, P_{2}\right.$ and $P_{3}$ ) during the mechanism work (Figs. 7 and 8 ). The driving wheel drives (by changing the angle $\varphi$ ) the slottedlink mechanism, which leads to changes in the angle of wing rotation $(\beta)$ and wing flapping $(\alpha)$ according to the diagram 6. Figure 6 contains additional points (on the $\mathrm{x}$ axis) that show the specific sequence of entomopter wing motion from Fig. 5. Angle $\varphi_{1}$ and $\varphi_{5}$ correspond to the position in Fig. 5a, angle $\varphi_{2}$ to the position in Fig. 5b, angle $\varphi_{3}$ to position in Fig. 5c, and angle $\varphi_{4}$ to position in Fig. $5 \mathrm{~d}$.
The values of angles $\alpha$ and $\beta$ can be described depending on the angle $\varphi$ with the following equations:

$\alpha(\phi)=\left(\alpha_{\max }-\alpha_{\min }\right)\left\{\begin{array}{l}\frac{\phi}{\phi_{3}-\phi_{2}} \mathrm{H}\left(\phi_{3}-\phi_{2}-\phi\right)+ \\ +\left[\mathrm{H}\left(\phi-\phi_{3}+\phi_{2}\right)-\mathrm{H}\left(\phi+\phi_{2}-\phi_{4}\right)\right]+ \\ +\frac{\phi_{5}-\phi_{2}-\phi}{\phi_{5}-\phi_{4}} \mathrm{H}\left(\phi+\phi_{2}-\phi_{4}\right)\end{array}\right\} \mathrm{H}(\phi) \mathrm{H}\left(\phi_{5}-\phi_{2}-\phi\right)-\alpha_{\max }$,

where: $\mathrm{H}()$ is the Heaviside function, $\phi=\phi_{b}-\left(\phi_{2}-\phi_{1}\right), \varphi_{b}$ is the current angle position $\varphi$ in range $<0,360^{\circ}>$,

$$
\beta(\phi)=\left(\beta_{\max }-\beta_{\min }\right)\left\{\begin{array}{l}
\frac{\phi}{\phi_{2}-\phi_{1}} \mathrm{H}\left(\phi_{2}-\phi_{1}-\phi\right)+ \\
+\left[\mathrm{H}\left(\phi-\phi_{2}+\phi_{1}\right)-\mathrm{H}\left(\phi+\phi_{1}-\phi_{3}\right)\right]++ \\
+\frac{\phi_{4}-\phi_{1}-\phi}{\phi_{4}-\phi_{3}} \mathrm{H}\left(\phi+\phi_{1}-\phi_{3}\right)
\end{array}\right\} \mathrm{H}(\phi) \mathrm{H}\left(\phi_{4}-\phi_{1}-\phi\right)-\beta_{\max },
$$

where: $\phi=\phi_{b}-\phi_{1}$.

\subsection{Simulation in SolidWorks}

Simulation tests were performed in Motion module of the SolidWorks software using a geometrical model (Fig. 3).

Fig. 11 Comparison of analytical results (trajectories of the characteristic points) with simulations in SolidWorks software

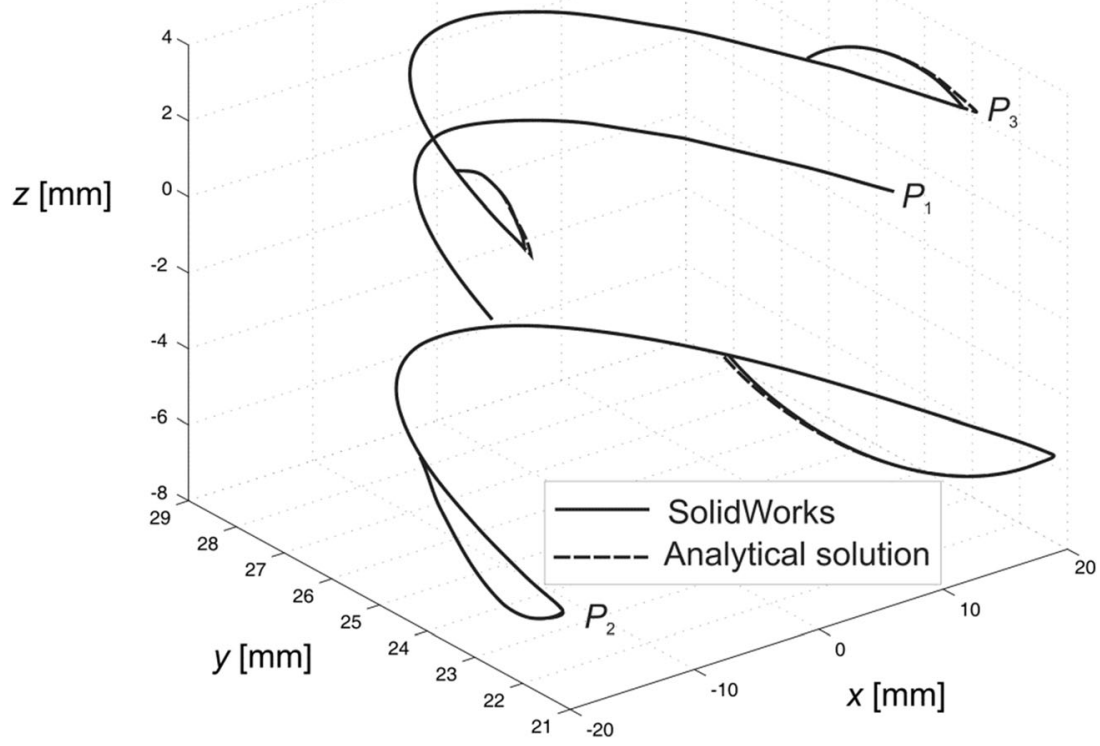


Fig. 12 Mechanism prototype

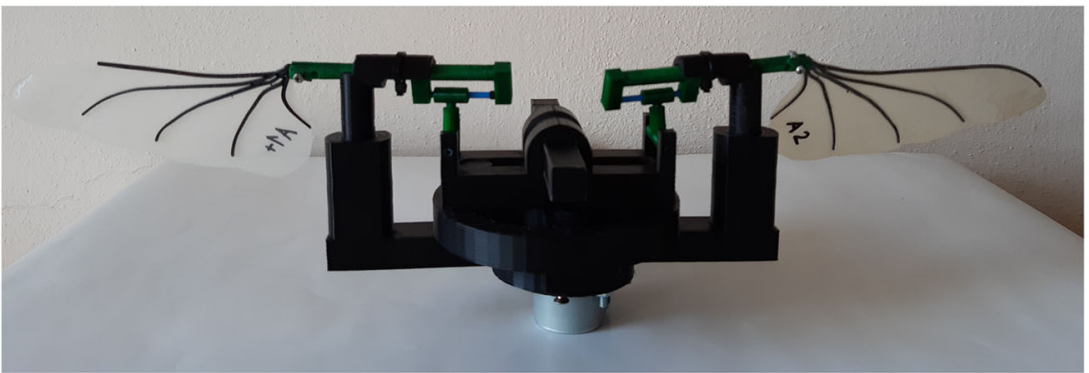

The system motion was forced by a virtual angular drive, which actuated the motion of the driving wheel. It was assumed that the mechanism would work at the constant speed of $25 \mathrm{rpm}$. The effect of other forces (e.g. gravitation) was neglected in the system.

Three additional points were added (see Fig. 9) to obtain the trajectories of the characteristic points $P_{1}$, $P_{2}$ and $P_{3}$ in the geometrical wing model. They allowed for the analysis of their spatial location during the mechanism work.

The system work (animation of the motion of the whole system) was documented for several seconds and the trace path of the added points was recorded (see Fig. 10).

In order to verify the work of the mechanism shown in Fig. 11, we compared the results obtained from the simulation performed in the SolidWorks software and those obtained from the relationship (1-3).

The comparative analysis presented in Fig. 11 shows that trajectories of the characteristic points (mimicking the insect's wing motion) obtained from the model prepared in SolidWorks software and those obtained from the kinematic model are very similar.

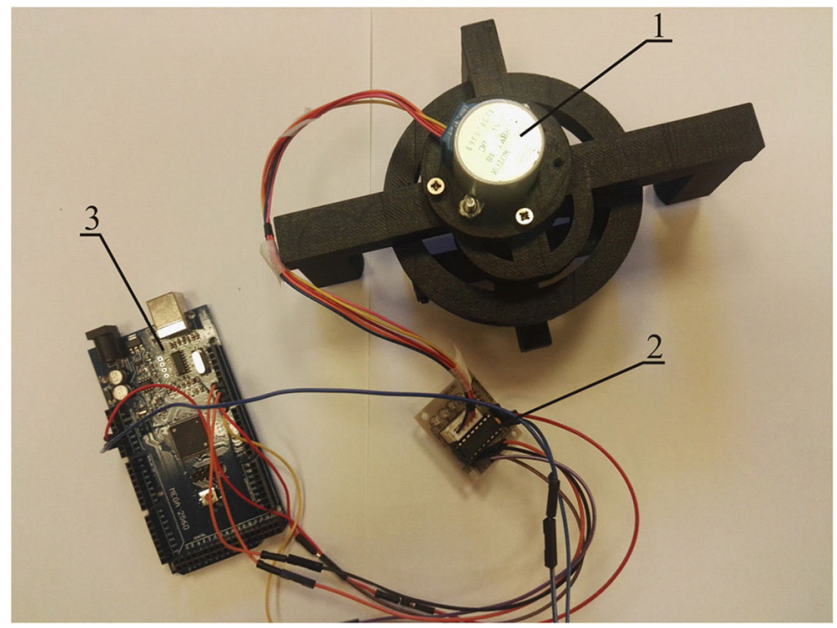

Fig. 13 Stepper motor (1), controller (2) and Arduino platform (3)

\subsection{Experimental verification of the mechanism's operation}

In order to verify the operation of the proposed model, a real object (Fig. 12) was made. Most of the elements were made of PLA material using the FDM (Fused Deposition Modeling) technique [42]. 3D printing was also used to make the wing frame, with which the foil imitating the wing was then welded. Normalized fasteners were used at the joints.

The system was driven using a stepper motor 28BYJ-48 (Fig. 13:1) with a 64:1 integrated gear reduction and a dedicated controller based on the ULN2003 system (Fig. 13:2). For motor control, an Arduino platform equipped with an ATmega2560 microcontroller was used (Fig. 13:3).

Sequences of subsequent wing positions during the mechanism's operation are shown in Fig. 14 (top view) and Fig. 15 (side view), while the work of the mechanism can be seen in the video under the link (http://www. imipkm.pcz.pl/wp-content/uploads/2016/08/New mechanism.wmv).

The experimental verification of the operation of the entomopter wing drive mechanism confirmed its correct operation and the possibility of using it in the design of entomopters or other flying objects.

In the case of the application of the presented solution for the real object it is sufficient to choose the system parameters in order to properly reflect the living organism. If it is assumed as in the study [18] that wing flapping angle $\alpha$ during a flight is $77^{\circ}$ (see Fig. 16), it suffices to modify the radius of the driving wheel of the slotted-link mechanism $(r)$ of the distance between the bilateral slider guide bushing and rocking lever guide bushing (b). According to (4), if the parameter $b$ $(7.4 \mathrm{~mm})$ is unchanged, the radius $r$ is $5.89 \mathrm{~mm}$, or if the radius $r$ is unchanged $(4.74 \mathrm{~mm})$, the distance $b(5.96 \mathrm{~mm})$ is modified.

Furthermore, if the angle of wing rotation $(\beta)$ was $90^{\circ}$ (Fig. 17), the design of wing lever guide would have to be modified.

A full comparative analysis of the model proposed in the work and the real object in terms of similarities and differences as well as the possibilities of controlling the object will be the subject of further work of the Authors. 

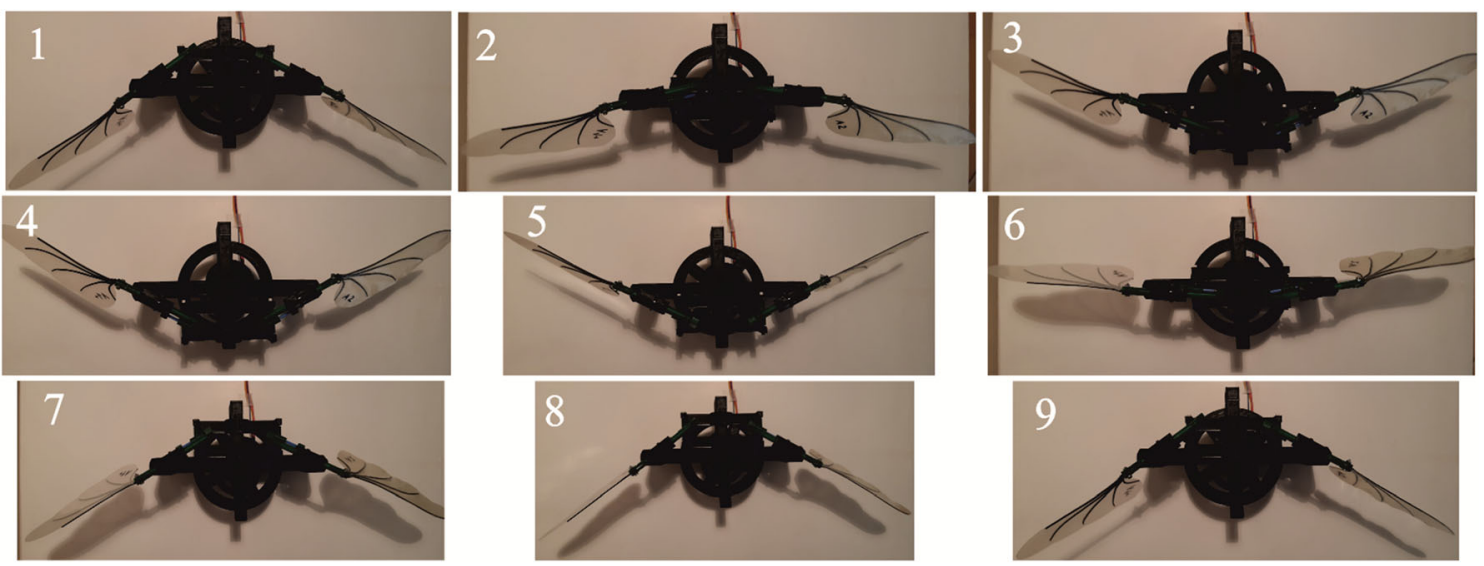

Fig. 14 Sequence of wing positions during mechanism operation (top view)

Fig. 15 Sequence of wing positions during mechanism operation (side view)
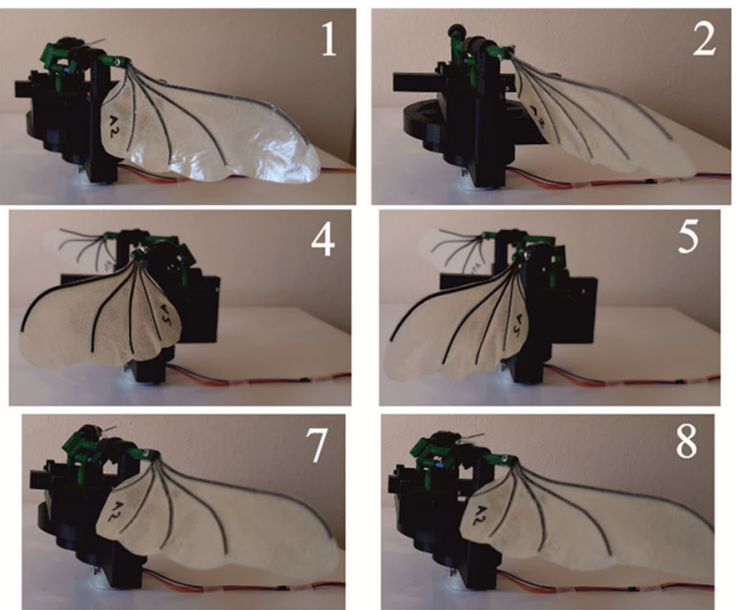

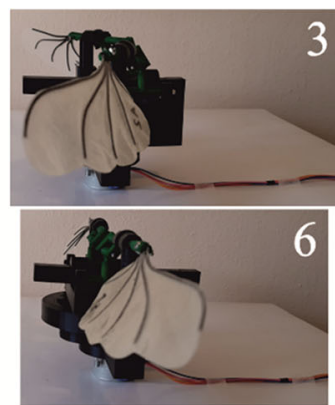

8

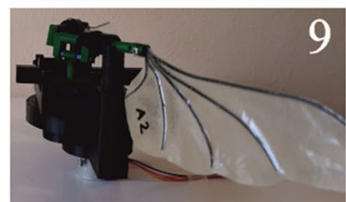

\section{Conclusions}

The design of the mechanism used for power transmission to the entomopter wings in order to perform a flapping motion and control the angle of attack of the wings was discussed in the paper. The kinematic scheme and the simulation model prepared in SolidWorks program for the proposed mechanism were shown. The results obtained on the basis of comparative analysis for both models proved that trajectories of the observed characteristic points are very similar. To verify the mechanism's operation, a prototype was built. Most of the elements were performed on a $3 \mathrm{D}$ printer, and the drive and control was implemented using a stepper motor with a suitable driver and an ATmega microcontroller. Experimental validation confirmed the correctness of the proposed solution in the scope of transmission of the drive from the motor to the wings of the entomopter by the mechanism of forcing the
Fig. 16 Range of changes in wing flapping angle $\alpha$ [18]
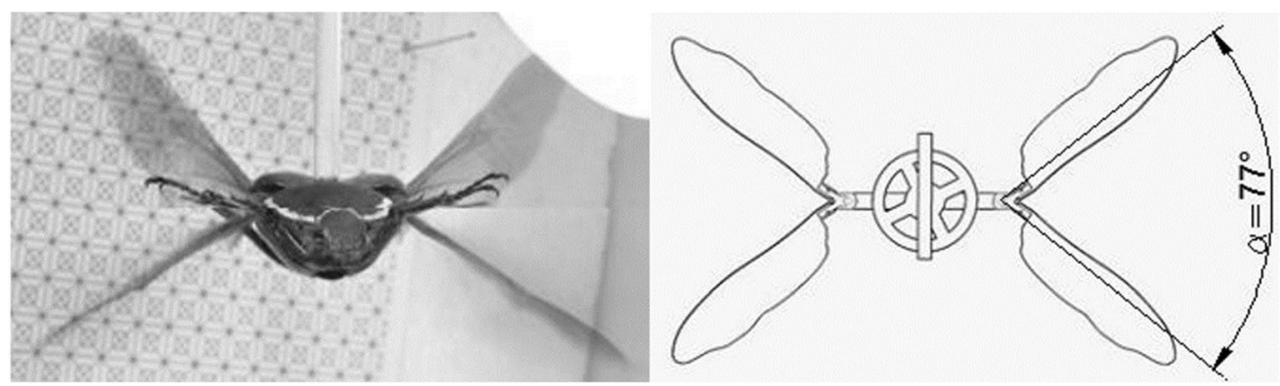


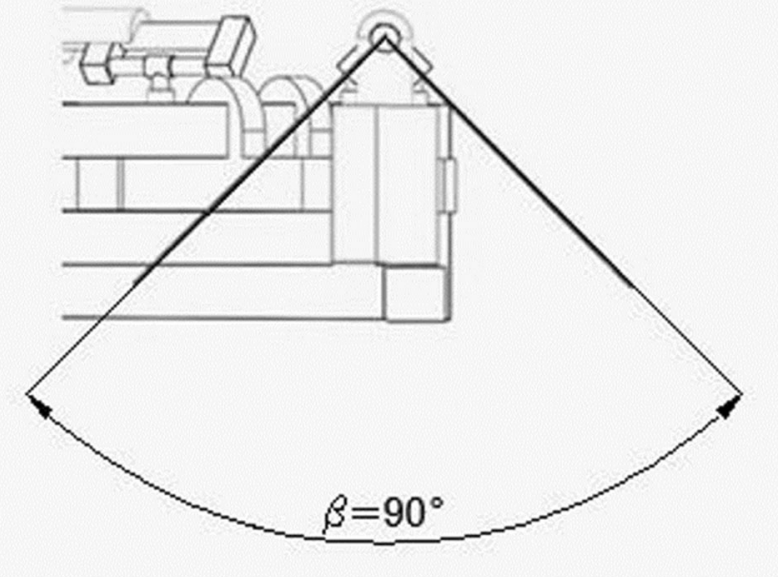

Fig. 17 Range of changes in the angle of wing rotation $(\beta)$ of the wings [18]

movement of the wings corresponding to the movement of the wings of the real insect (Melolontha melolontha).

The presented mechanism for power transmission to entomopter wings using the slotted-link and slider mechanism is characterized by simplicity and offers opportunity for miniaturization while ensuring reliable work at reduced demand for power to drive the mechanism. A technological advantage of the presented solution is the use of only one component between the slotted-link mechanism and the rocking lever of the wing in the form of a bilateral slider in order to perform flapping wing motion and change the angle of attack of the entomopter. Numerical simulations demonstrated that it is possible to design an entomopter model with the mechanism proposed in the study to perform basic insect wing movements in the range of actual flapping angles and angles of attack.

The considered model of the mechanism is under patent protection [43].

Acknowledgments This research was supported by the Ministry of Science and Higher Education in 2020, Warsaw, Poland.

Open Access This article is licensed under a Creative Commons Attribution 4.0 International License, which permits use, sharing, adaptation, distribution and reproduction in any medium or format, as long as you give appropriate credit to the original author(s) and the source, provide a link to the Creative Commons licence, and indicate if changes were made. The images or other third party material in this article are included in the article's Creative Commons licence, unless indicated otherwise in a credit line to the material. If material is not included in the article's Creative Commons licence and your intended use is not permitted by statutory regulation or exceeds the permitted use, you will need to obtain permission directly from the copyright holder. To view a copy of this licence, visit http://creativecommons.org/licenses/by/4.0/.

\section{References}

1. Abas MFB, Rafie ASBM, Yusoff HB, Ahmad KAB (2016) Flapping wing micro-aerial-vehicle: kinematics, membranes, and flapping mechanisms of ornithopter and insect flight. Chin J Aeronaut 29(5:1159-1177

2. Geisler T, Rosikoń P, Sochacki W, Topczewska S (2014) Functional and structural analysis of wing folding mechanism based on cockchafer (Melolontha melolontha). Acta Mechanica et Automatica 8(3):129-135

3. George RB, Colton MB, Mattson CA, Thomson SL (2012) A differentially driven flapping wing mechanism for force analysis and trajectory optimization. Int J Micro Air Vehicles 4(1):31-49

4. Venkiteswaran V K, Su H. Optimization of mechanism design of flapping wing MAV. AIAA SciTech, 2014, National Harbor, Maryland

5. Anderson M L, Sladek N J, Cobb R.G.. Design, Fabrication, and Testing of an Insect-Size MAV Wing Flapping Mechanism. 49th AIAA Aerospace Sciences Meeting Including the New Horizons Forum and Aerospace Exposition, 2011, Orlando, Florida

6. Yang LJ, Hsu CK, Ho JY, Feng CK (2007a) Flapping wings with PVDF sensors to modify the aerodynamic forces of a micro aerial vehicle. Sensors Actuators A Phys 139(1-2):95-103

7. Galiński C, Żbikowski R (2005) Insect-like flapping wing mechanism based on a double spherical scotch yoke. J Royal Socc Interface 2(3):223-235

8. Keshavan J, Wereley N M. Design and Development of a High Frequency Biologically Inspired Flapping Wing Mechanism. AIAA online proceedings, 2007, AIAA 2007-1789

9. Gerdes JW, Cellon KC, Bruck HA, Gupta SK (2013) Characterization of the mechanics of compliant wing designs for flapping-wing miniature air vehicles. Exp Mech 53(9):1561-1571

10. Bejgerowski W, Ananthanarayanan A, Mueller D, Gupta S K. Integrated product and process design for a flapping wing drive mechanism. J Mech Des, 2009, 131(6)

11. Anderson M L. Design and Testing of Flapping Wing Control for a Micro Air Vehicle. AIAA Guidance, Navigation and Control Conference, 2011, Portland, Oregon

12. Fenelon MAA, Furukawa T (2010) Design of an active flapping wing mechanism and micro aerial vehicle using a rotary actuator. Mech Mach Theory 45(2):137-146

13. McIntosh SH, Agrawal SK, Khan Z (2006) Design of a Mechanism for biaxial rotation of a wing for a hovering vehicle. Trans Mechatronics 11(2):145-153

14. Oppenheimer M W, Sigthorsson D O, Weintraub I E, Smith T J, Dawson J C, Doman D B. Development of Flapping Wing Mechanism That Can Produce Lift Greater Than Weight. AIAA Guidance, Navigation and Control Conference, 2013, Boston, MA

15. Chen D, Yin J, Chen K, Zhao K, Zhang B (2014) Prototype design and experimental study on locust air-posture righting. J Bionic Eng $11: 459-468$

16. Madangopal R, Khan ZA, Agrawal SK (2005) Biologically inspired design of small flapping wing air vehicles using four-bar mechanisms and quasi-steady aerodynamics. J Mech Des 127(4): 809-816

17. Dawson J C, Huang P G. Figure-8 Flapping Micro Air Vehicle. AIAA Aerospace Sciences Meeting including the New Horizons Forum and Aerospace Exposition, 2011, Orlando, Florida

18. Geisler T. Observation and measurements of wing parameters of the selected beetle spacies and the design of a mechanism structure implementing a complex wing movement. Int J Appl Mechan Eng, 2016, 21(4), 837-847

19. Nguyen QV, Park HC, Goo NS, Byun D (2010) Characteristics of a Beetle's free flight and a flapping-wing system that mimics beetle flight. J Bionic Eng 7:77-86 
20. Lesage F, Hamel N, Huang X, Yuan W, Khalid M, Zdunich P. Initial investigation on the aerodynamic performance of flapping wings for nano air vehicle. Technical Memorandum DRDC Valcatier TM 2007-550 (report), 2008

21. Raney D L, Slominski E C. Mechanization and Control Concepts for Biologically Inspired Micro Aerial Vehicles. AIAA online proceedings, 2003, AIAA 2003-5345

22. Yang L, Hsua C, Hoa J, Feng C (2007b) Flapping wings with PVDF sensors to modify the aerodynamic forces of a micro aerial vehicle. Sensors Actuators A Phys 139:95-103

23. Nguyen VQ, Syaifuddin M, Park HC, Byun DY, Goo NS, Yoon KJ (2008) Characteristics of an insect-mimicking flapping system actuated by a unimorph piezoceramic actuator. J Intell Mater Syst Struct 19(10): 1185-1193

24. Wood R J. Liftoff of a 60mg flapping-wing MAV. IEEE/RSJ Int. Conf. on Intelligent Robots and Systems, 2007, Las Vegas, USA, 1889-1894

25. Wood R. Fly, robot, Fly IEEE Spectrum, 2008, 45(3), 25-29

26. Whitney J P. Design and performance of insect-scale flapping-wing vehicles. PhD thesis, 2012, Harvard University

27. Wu J, Qiu J, Zhang Y (2017) Automated kinematics measurement and aerodynamics of a bioinspired flapping rotary wing. J Bionic Eng 14:726-737

28. de Croon GCHE, De Clercq KME, Ruijsink R, Remes B, de Wagter C (2009) Design, aerodynamics and vision-based control of the DelFly. J Micro Air Vehicles 1(2):71-97

29. Lentink D, Jongerius S R, Bradshaw N L. The Scalable Design of Flapping Micro-Air Vehicles Inspired by Insect Flight, SpringerVerlag, Berlin, 2009

30. Hu Z, Cheng B, Deng X. Lift Generaton and Flow Measurement of a Robotic Insect. AIAA online proceedings, 2011, AIAA 20111311

31. Sahai R, Galloway K C, Karpelson M, Wood R J. A Flapping-Wing Micro Air Vehicle with Interchangeable Parts for System
Integration Studies. IEEE/RSJ International Conference on Intelligent Robots and Systems, 2012, Vilamoura, Portugal, 501506

32. Ratti J, Vachtsevanos G (2010) A biologically-inspired micro aerial vehicle: sensing, modeling and control strategies. J Intell Robot Syst 60:152-178

33. Kelfer J W, A Figure eight wing drive, Patent US 4793573, 1998

34. Smith MJC (2003) Wing-drive mechanism and vehicle employing same. Patent US 6568634:B2

35. Smith MJC (2004) Wing-drive mechanism and vehicle employing same. Patent US 6783097:B1

36. Sinclair PL (2007) Motion assisting apparatus. Patent US 7204455 : B2

37. University Of Delaware, Mechanism for biaxial rotation of a wing and vehicle containing such mechanism, Patent US 7651051 B2, 2010

38. Therriault C (2001) Wing movement for ornithopters and apparatus of the like. Patent US 6227483:B1

39. Sharp Kabushiki Kaisha, Rising and moving apparatus and manufacturing method thereof, Patent US 7219855 B2, 2007

40. Georgia Tech Research Corporation, Hovering and gliding multiwing flapping micro aerial vehicle, Patent US 20130320133 A1, 2013

41. Cekus D, Posiadala B, Warys P (2014) Integration of modeling in SolidWorks and Matlab/Simulink environments. Archive Mech Engineering 61(1):57-74

42. Gebhardt A, Hötter J. Additive Manufacturing. 3D Printing for Prototyping and Manufacturing, Hanser, Munich, 2016

43. Sochacki W, The power transmission mechanism to the entomopter's wings, Patent PL 232749 B1, 2019 (in Polish)

Publisher's Note Springer Nature remains neutral with regard to jurisdictional claims in published maps and institutional affiliations. 